\title{
PROPUESTA CURRICULAR QUE OFRECE LA UNIVERSIDAD DE COSTA RICA PARA LA FORMACIÓN DE PROFESORES DE CIENCIAS
}

\section{Master Leda María Roldán Santamaría'}

RESUMEN: El presente artículo recoge los resultados de una investigación realizada para determinar las necesidades de cambio en el plan de estudios de formación de profesores y bachilleres en la enseñanza de las ciencias naturales que se gradúan en la Universidad de Costa Rica. Al final se presenta una propuesta curricular para lograr la actualización del mismo.

ABSTRAC: This article is the report of the investigation about of the Academic program of Sciences' teacher in the University of Costa Rica. That program was presented in 1990 and in this moment it recoated changes. Finally it present the new project for the academy program for Natural Science's teacher.

Palabras clave: ENSEÑANZA DE LAS CIENCIAS/ INVESTIGACIÓN EN ENSEÑANZA DE LAS CIENCIAS/ FORMACIÓN DE PROFESORES DE CIENCIAS/

\section{Introducción}

El programa de formación de profesores de Ciencias, Física, Química y Biología, que se ofrece y desarrolla actualmente en la Universidad de Costa Rica se aprobó en 1990. Después de que éste se puso en vigencia, el Ministerio de Educación Pública revisó y modificó los programas de enseñanza de la Educación Media. Por otra parte, la ciencia y la tecnología han experimentado avances significativos y han surgido nuevas teorías en el campo de la enseñanza y aprendizaje de las ciencias.

La formación de profesores de ciencias para la Educación General Básica y Diversificada es, para la Universidad de Costa Rica, una oportunidad de apoyar el desarrollo de la educación costarricense y de favorecer el avance de la ciencia y la tecnología. Es apreciable que los profesores de ciencias del nivel de Enseñanza Media que logran despertar en sus estudiantes el interés por el campo científico, logren a su vez una alta posibilidad de que esos jóvenes lleguen a ser adultos que favorezcan el desarrollo de la ciencia y la tecnología en nuestro país.

\footnotetext{
1 Profesora de Enseñanza Media de la Universidad de Costa Rica. Bachiller y Licenciada en Física de la Universidad de Costa Rica. Master en Planificación Curricular de la Universidad de Costa Rica. Profesora en las cátedras de servicio de la Escuela de Física y de Estudios Generales y de cursos de Física y de Educación para estudiantes de la carrera de Enseñanza de las Ciencias de la Universidad de Costa Rica. Iroldan@cariari.ucr.ac.ch
} 
La necesidad de fortalecer la enseñanza de las ciencias y de mejorar la calidad de la formación de los estudiantes costarricenses en este campo, motiva a revisar y actualizar el plan de formación de bachilleres en la Enseñanza de las Ciencias. Como comentara en una reciente conferencia, León Lederman, el premio Nóbel de Física 1988: “. . .Ante la revolución que estamos viviendo, es necesario cambiar la forma en que percibimos las nuevas tecnologías y aplicaciones de la ciencia ...La nueva forma de enseñar ciencias consiste también en enseñar a los maestros cómo enseñar ciencias." (CENAT, Costa Rica, 2002)

Ante la necesidad de actualizar los programas de formación de profesores de Ciencias, Física, Química y Biología que se gradúan en la Universidad de Costa Rica, se revisaron los objetivos y contenidos de los cursos que se incluyen en ese plan de estudios, tanto en el área científica como pedagógica. Esa revisión se llevó a cabo por medio de consultas a diferentes informantes en el área de la enseñanza de las ciencias y mediante revisión de fuentes bibliográficas de los planes anteriores a 1998, en la enseñanza de las ciencias. De los hallazgos de la investigación se observa que existe necesidad de generar un plan que contemple una estructura vertical en cuanto al nivel de profundidad de la formación y horizontal en cuanto a los diferentes campos del conocimiento, el cual, debe abarcar dicha formación.

De esas consultas y revisiones se plantean hallazgos significativos, los cuales se presentan en la siguiente tabla, relacionados con los objetivos de la investigación realizada:

Tabla 1: Hallazgos significativos logrados según cada objetivo.

\section{Objetivos}

Confrontar el perfil profesional vigente del profesor de ciencias con la propuesta programática de los cursos que ofrece el plan de estudios.

Valorar los contenidos pedagógicas de la carrera de Es necesario revisar los contenidos y metodología del bachillerato según el perfil núcleo pedagógico con miras a actualizarlo. profesional Determinar los contenidos propios de las disciplina de las Ciencias que ofrece el actual plan de formación de profesores de esta materia.

\section{Hallazgos significativos}

Debe fortalecerse el estímulo a la investigación, educativa y científica, para que esta sea consecuente con las exigencias actuales en cuanto a las nuevas teorías pedagógicas en la enseñanza de las ciencias.

El plan de estudios vigente requiere de una actualización en los conceptos científicos para que así, haya coherencia con el avance científico y tecnológico de nuestro país. 


\begin{tabular}{|c|c|}
\hline $\begin{array}{l}\text { Identificar el nivel de } \\
\text { profundidad en el abordaje de } \\
\text { los conceptos científicos que } \\
\text { se cubren en dicho plan, de } \\
\text { acuerdo con el avance científico } \\
\text { y tecnológico. }\end{array}$ & $\begin{array}{l}\text { El nivel de cada uno de los cursos que se ofrece en el } \\
\text { plan de estudios es el adecuado. Debe velarse por no } \\
\text { modificar dicho nivel de complejidad, ya que se } \\
\text { considera que el profesor de ciencias debe conocer más } \\
\text { de lo que contiene el programa de enseñanza con el que } \\
\text { va a trabajar. Es necesario incluir cursos que brinden los } \\
\text { temas que se encuentran ausentes en dicho plan. }\end{array}$ \\
\hline $\begin{array}{l}\text { Contrastar los conceptos } \\
\text { científicos que le ofrece el plan } \\
\text { de estudios con los que se } \\
\text { requieren en los programas de } \\
\text { estudios, vigentes, del MEP. }\end{array}$ & $\begin{array}{l}\text { Es necesario revisar los cursos optativos con la idea de } \\
\text { que se actualice dicha lista y se ofrezcan más opciones, } \\
\text { con el propósito de cubrir con ellos los contenidos } \\
\text { faltantes en el plan de estudios vigente. }\end{array}$ \\
\hline $\begin{array}{l}\text { Identificar los cambios que } \\
\text { requiere el actual plan de } \\
\text { estudios para presentar una } \\
\text { propuesta de malla curricular } \\
\text { para la formación de profesores } \\
\text { de Ciencias Naturales. }\end{array}$ & $\begin{array}{l}\text { Es necesario ajustar los cursos } \\
\text { área pedagógica. Se les debe } \\
\text { plan de estudios quede equilibr } \\
\text { cumpla con los requisitos y corr }\end{array}$ \\
\hline $\begin{array}{l}\text { Diseñar una propuesta de } \\
\text { currículo acorde con la realidad } \\
\text { nacional y el avance científico y } \\
\text { tecnológico. }\end{array}$ & $\begin{array}{l}\text { Una propuesta de plan de estudios debe considerar las } \\
\text { nuevas teorías psicopedagógicas que favorecen el } \\
\text { proceso de enseñanza y de aprendizaje. Debe incluir los } \\
\text { conocimientos sobre los avances científicos y } \\
\text { tecnológicos con miras a formar a un profesor con una } \\
\text { visión pedagógica según las tendencias actuales. }\end{array}$ \\
\hline
\end{tabular}

A partir de esos hallazgos significativos, surgieron las siguientes recomendaciones:

1. Las autoridades de la Universidad de Costa Rica en general, de la Escuela de Formación Docente y de las unidades académicas de la Facultad de Ciencias, en particular deben considerar los objetivos que se persiguen en la formación de un bachiller en la enseñanza de las ciencias naturales. El perfil satisface las condiciones del plan vigente; pero para su actualización, es necesario revisarlo y hacer una propuesta curricular que comprenda todos estos aspectos, y que permita formar un profesor conocedor de las demandas del sistema educativo y de la sociedad costarricense.

2. La Comisión Compartida de Enseñanza de las Ciencias en la Universidad de Costa Rica, deberá proponer una serie de cambios para cubrir los ". . .conceptos propios de la disciplina " y lograr un ". . . nivel en que se abordan los conceptos científicos". Para lograrlo es necesario revisar la lista de cursos optativos y actualizarla, para que de esta forma, los estudiantes puedan optar por cursos que le ofrezcan una formación más amplia. Es necesario incluir cursos obligatorios en el campo de la Geología de Costa 
Rica y la Fisiología Humana, pues asía la formación del docente estará de acuerdo con su nivel profesional.

3. Para las autoridades que administran dicho plan, es recomendable hacer una revisión de los contenidos científicos y su distribución dentro del programa de estudios. Con esto, se puede garantizar el avance y la calidad en la formación de los profesores.

4. Conviene que la Escuela de Formación Docente revise los contenidos del núcleo pedagógico; esto con miras a formar un profesor con una visión pedagógica que implique las tendencias actuales y que satisfaga necesidades de las nuevas políticas educativas costarricenses; entre las que se considera que el aprendizaje significativo integra lo aprendido a la estructura cognitiva, y puede aplicarse a situaciones y contextos distintos a los que se aprendieron inicialmente. Además, según esta visión, los conocimientos se conforman en redes de significados más amplios y complejos, lo cual posibilita que puedan ser recordados con más facilidad. De acuerdo con las teorías del constructivismo, los estudiantes deben tener una fuerte conceptualización del mundo que los rodea, y deben haber construido bases firmes que les permitan continuar construyendo los nuevos conceptos científicos con ayuda del uso que tales conceptos tienen en ese entorno. Es conveniente que el docente le presente a su alumno condiciones adecuadas que lo motiven a continuar construyendo nuevos conceptos.

5. Para atender las recomendaciones antes mencionadas, se presentó una propuesta curricular que las tomó en cuenta. El plan de estudios que se estableció satisface las necesidades de los estudiantes y contempla el avance científico y tecnológico con miras a lograr una mejor formación del profesor de Ciencias Naturales.

Los resultados y las recomendaciones expuestas son producto de un análisis exhaustivo de la información. Como resultado de la investigación, se presentó una propuesta de modificación del plan de estudios de bachillerato en la Enseñanza de las Ciencias Naturales, acompañada de un esquema del mapa curricular. Esta se elaboró considerando los contenidos científicos, pedagógicos y humanísticos que debe tener el profesor de ciencias naturales, tanto para cubrir los programas que de esa especialidad tiene el Ministerio de Educación Pública, como para tener en las aulas de secundaria un profesor investigador que desee innovar en la enseñanza de las ciencias. 


\section{Presentación de la propuesta}

El diseño curricular que respalda esta propuesta, parte de los siguientes conceptos básicos:

- Enfoque curricular: Según la Vicerrectoría de Docencia, la práctica curricular de la Universidad de Costa Rica se caracteriza por el predominio del enfoque académico y por la diversidad en los diseños de los planes de estudio. En estos se manifiestan diversos niveles de articulación entre la teoría y la realidad. Sin embargo, desde finales de la década del 70 , se observa una tendencia creciente a dar énfasis al hacer sobre el comprender y el explicar, producto de la influencia de los enfoques tecnológicos. (Vic. Docencia, 1995; p. 15)

- Plan de estudios: Persigue la presentación de un orden lógico de factores que influyen en la formación profesional e integral de una persona, con características específicas para las necesidades sociales y económicas. Es adecuado que incluya el perfil profesional y las expectativas de desenvolvimiento social que se espera de ese profesional. En ese ordenamiento, se incluyen:

- Cursos humanísticos, pedagógicos y científicos para la construcción del conocimiento sobre un campo epistemológico específico.

- Créditos de cada curso.

- Ubicación de los cursos de manera que se cumplan los requisitos y correquisitos adecuadamente.

La aprobación de esos cursos conduce al estudiante a la obtención de un título universitario que lo acredita para ejercer la profesión que eligió.

- Visión educativa de la función del profesor de ciencias: La Educación es el proceso de transformación, consolidación, reacción y recreación de la cultura, y así debe visualizarlos el profesor de Ciencias. Como se planteó en la Declaración de Santo Domingo sobre la Ciencia para el siglo XXI, una nueva visión y un marco de acción sobre educación y formación científica y tecnológica: ". . . La renovación de la enseñanza de la ciencia y la tecnología, por vías formales e informales, debe apuntar también a promover la comprensión pública de la ciencia y la tecnología como parte de la cultura." (UNESCO, 1999; p. 1) 
- Currículo para la formación de docentes en la Enseñanza de las Ciencias: Este currículum se fundamenta en principios filosóficos que visualizan al ser humano como un ser capaz de modificarse de acuerdo con su entorno y de utilizar los conocimientos construidos para transformar el ambiente de trabajo en beneficio de sus estudiantes; con principios éticos que lo motivan a vivir dignamente y a propiciar esos principios en sus estudiantes y capaz de utilizar los principios epistemológicos para lograr que sus alumnos se desarrollen como seres individuales, pero con sentido de socialización.

- Concepto de Aprendizaje: Este no es una simple acumulación de información, sino una integración de los nuevos conocimientos con base en los que adquirió y construyó anteriormente; de manera que, si es preciso, modifique la organización de estos últimos. La experiencia profesional lleva a considerar que la integración y reorganización de conocimientos podría afectar los esquemas conceptuales, los conocimientos sobre procedimientos y los conocimientos sobre las condiciones en las cuales usar lo que ya se sabe, o sea, las condiciones en las se puede utilizar ese aprendizaje.

\subsection{Fases del diseño curricular}

Todo diseño curricular tiene varias fases, las fundamentales se mencionan a continuación:

1. Diagnóstico.

2. Diseño.

3. Presentación curricular.

4. Ejecución como etapa de prueba.

5. Evaluación de resultados.

La propuesta cubre las tres primeras fases de su ejecución, porque los objetivos de la investigación no contemplaron desarrollar las otras dos; ya que estas le corresponden a la Escuela de Formación Docente, unidad académica que tiene la capacidad y experiencia para llevarlas a cabo. Sin embargo, se presentan sugerencias para cumplir posteriormente con las etapas. 
Como plan de estudios propuesto, se piensa en su administración y evaluación desde la posición de que todo plan tiene un seguimiento evaluativo que permite mejorarlo sobre la marcha.

\subsection{Esquema que respalda esta propuesta}

La propuesta considera los entes involucrados en la formación del profesor de Ciencias Naturales y los aspectos generales que se tienen presentes para la formación del profesional que aquí se menciona. La Universidad de Costa Rica es la responsable de este plan de estudio; el cual, es administrado por la Escuela de Formación Docente y las escuelas de la Facultad de Ciencias. Son estas entidades las llamadas a la revisión de planes de estudio y su actualización.

La revisión de planes y programas obliga a tener en cuenta el avance de la Ciencia y la Tecnología y las nuevas teorías en lo que a formación del profesor de Ciencias se refiere. Por lo que esta propuesta debe contemplar esos campos.

La formación del profesional en la enseñanza de las Ciencias se encuentra relacionada con el Ministerio de Educación Pública como ente contralor de la Educación Costarricense. Junto con las instituciones públicas, pertenecientes al Ministerio, las instituciones de Educación Media en la categoría de privadas y semioficiales, son los mayores empleadores de los docentes graduados.

A continuación, se presenta un esquema que representa el conjunto de interrelaciones antes expuesto; en el que el núcleo central es el programa de Bachillerato en la Enseñanza en las Ciencias Naturales. Hacia él, convergen los aspectos externos e internos que lo nutren. 


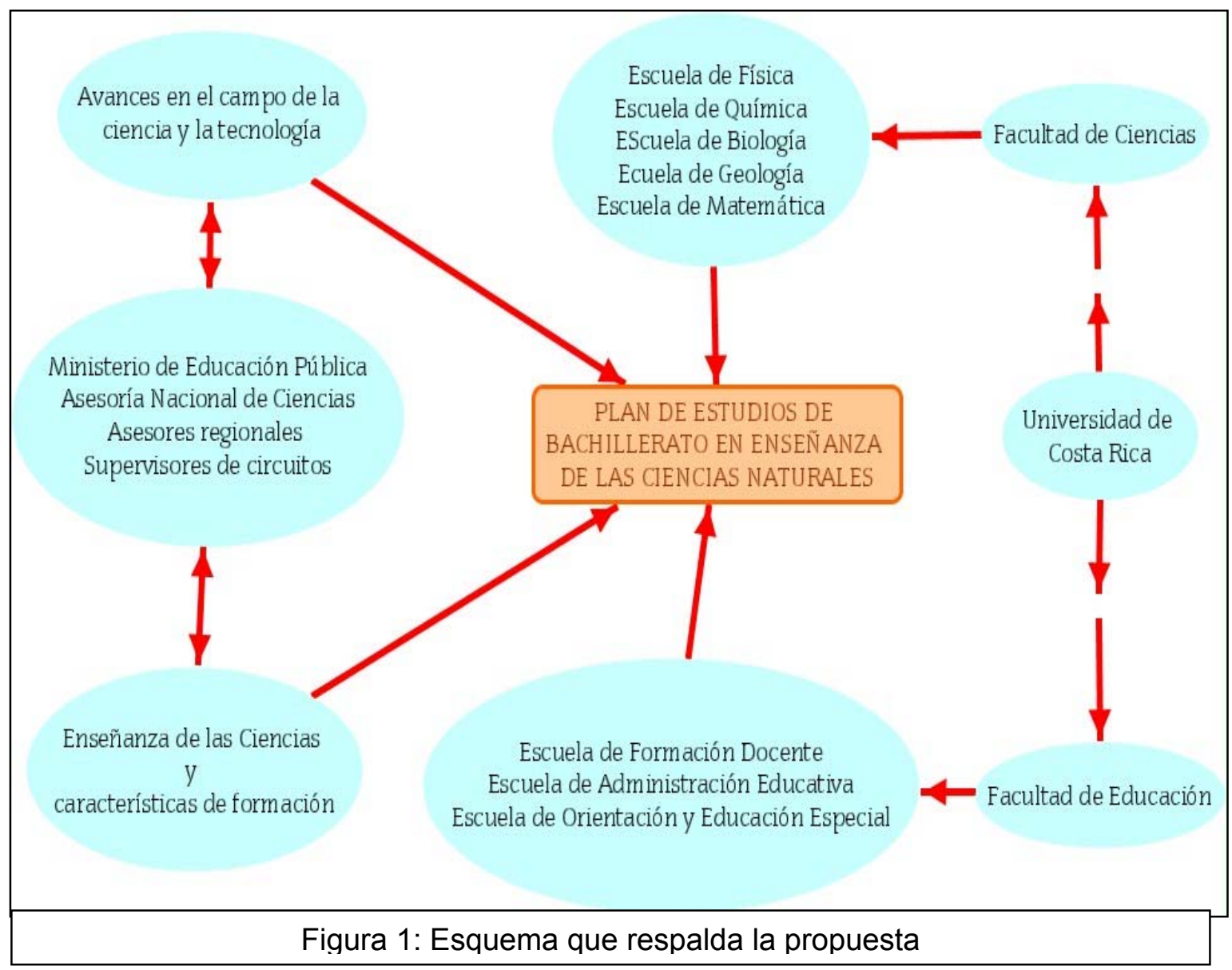

Los aspectos internos son la base del programa de formación. La Universidad de Costa Rica se apoya en la Facultad de Educación y en la Facultad de Ciencias como unidades de formación de este profesor. Cada una de esas facultades cuenta con el apoyo de sus unidades académicas, que son las que ofrecen los cursos que corresponden al plan de estudios. Adicional a ellas, existen otras unidades académicas que fortalecen dicha formación en el campo humanístico integral.

Los aspectos externos son aportados por los empleadores y el Ministerio de Educación Pública, junto con sus dependencias descentralizadas y la sociedad en general. También, se consideran las instituciones privadas y semioficiales que solicitan los servicios de estos profesionales. La sociedad expresa sus necesidades y demandas generales y los aportes sobre los avances de la ciencia y la tecnología, que se toman en cuenta para la formación del profesor y las características que esa sociedad espera del profesional. 
Todos estos aspectos contribuyen a presentar una propuesta de formación del profesor de ciencias acorde con las necesidades y demandas actuales.

\subsection{Perfil profesional}

El Centro de Evaluación Académica (CEA) define el perfil profesional como “. . . el conjunto de características que debe presentar un profesional en términos de conocimientos, habilidades y actitudes, para ejercer adecuadamente las funciones en el campo de su especialidad." (CEA, 1991; p. 3)

El actual plan de Bachillerato en la Enseñanza de las Ciencias presenta un perfil profesional dividido en tres áreas: humanística, pedagógica y científica.

La presente propuesta persigue establecer los lineamientos que definen el perfil profesional del profesor de Ciencias, de acuerdo con una visión moderna, coherente con las necesidades del sistema educativo costarricense y con una visión profesional que corresponda a los tiempos actuales.

El diagnóstico evidencia la necesidad de formar un profesional actualizado y con los conocimientos psicopedagógicos apropiados para la población que debe atender, por lo que el perfil profesional debe fortalecerse.

En esta propuesta se define un perfil de acuerdo con las nuevas tendencias educativas expresadas en el informe Delors (Delors, 1999; p. 25), al considerar los cuatro pilares de la educación.

La Escuela de Formación Docente, en la evaluación del núcleo pedagógico, consideró esos cuatro pilares para definir el perfil del egresado de este núcleo (Carvajal, 1999; 59). Estos ejes se toman en cuenta en esta propuesta.

Con este perfil, se espera que el bachiller en Enseñanza de las Ciencias Naturales reúna las siguientes características: 


\section{Aprender a conocer.}

- Reconoce las características sociales, culturales, políticas y económicas de la realidad costarricense.

- Establece relaciones entre las teorías pedagógicas y el contexto educativo del aula.

- Considera el análisis de los fundamentos filosóficos, antropológicos, políticos y culturales de las teorías pedagógicas que orientan su labor docente.

- Se actualiza con respecto a las nuevas teorías pedagógicas, metodológicas y didácticas.

- Consolida su formación psicopedagógica para el nivel de estudiantes que deberá atender en su desarrollo profesional.

- Fundamenta su labor docente en principios teóricos de enseñanza y aprendizaje.

- Desarrolla destrezas de autoaprendizaje.

- Fortalece su conocimiento del campo de la Física, la Química, la Biología y las áreas afines.

\section{Aprender a hacer.}

- Integra los conocimientos científicos, destrezas y habilidades propias de su disciplina en la planificación, ejecución y evaluación de su labor docente.

- Hace uso de la autoevaluación como un recurso para retroalimentar su labor.

- Establece relaciones entre la teoría y la práctica pedagógica para innovar en el aula.

- Desarrolla actividades docentes, investigativas y de acción social.

- Utiliza la investigación y la acción social para fortalecer su labor docente.

- Utiliza estrategias metodológicas de acuerdo con criterios curriculares.

- Ajusta los programas y las actividades de enseñanza y aprendizaje a las necesidades y expectativas de los educandos.

- Estimula en sus estudiantes la investigación, tanto en el área social como en el área científica.

- Hace uso de los recursos tecnológicos a su alcance para mejorar el proceso de enseñanza y aprendizaje.

- Atiende las necesidades curriculares y extracurriculares de sus estudiantes con necesidades educativas especiales.

\section{Aprender a vivir.}

- Promueve su participación y la de los educandos en experiencias interdisciplinarias. 
- Participa con sus colegas y estudiantes en actividades de bien común.

- Estimula en sus educandos una actitud de respeto hacia el ambiente.

- Hace evidente su interés y responsabilidad por la preservación de los recursos naturales.

- Promueve relaciones de respeto entre colegas, estudiantes, padres de familia y miembros de la comunidad.

\section{Aprender a ser.}

- Se interesa por ejercer de manera autónoma y responsable la misión docente en el marco institucional y comunitario.

- Se manifiesta como líder para conducir el cambio y la innovación educativa.

- Participa en las actividades que conducen a un mayor desarrollo profesional.

- Estimula en el educando el desarrollo del pensamiento crítico ante los cambios científicos y tecnológicos.

- Hace evidente su formación humanística y cultural, que responde a los lineamientos establecidos por la Universidad de Costa Rica.

- Es creativo en el diseño, ejecución y evaluación de su tarea docente.

- Desarrolla una actitud crítica y científica que promueve la resolución de problemas.

El bachiller en la Enseñanza de las Ciencias debe estimular, mediante la enseñanza y el aprendizaje de la disciplina, la construcción de conceptos en las Ciencias Naturales que permiten derivar conclusiones útiles para enfrentar la vida con actitudes creativas y racionales, que favorecen el logro de destrezas, habilidades, valores, actitudes, y principios conceptuales, que contribuyen a su vez, con el desarrollo integral del individuo, así como el manejo de nuevas tecnologías y el uso crítico de la información.

La enseñanza de las ciencias también debe promover la práctica de procedimientos, valores y actitudes propios del quehacer científico; tales como la autodisciplina, la responsabilidad, el respeto y la tolerancia, el aprecio por la comprobación de los hechos, la resolución de problemas y la objetividad en la búsqueda de explicaciones razonables, necesarias para comprender la realidad personal y ubicarse en el contexto ambiental.

La educación científica debe propiciar una relación más justa entre los seres humanos y la naturaleza y promover la utilización adecuada de los elementos naturales, requisito 
indispensable para la supervivencia de la humanidad, su calidad de vida y el desarrollo sostenible de nuestra región.

\section{Objetivos del plan de estudios de Bachillerato en Enseñanza de las Ciencias}

El plan de Bachillerato en la Enseñanza de las Ciencias proporcionará al estudiante los medios necesarios para:

1. Conocer los principios de las teorías científicas para desenvolverse en el nivel de las Ciencias Básicas en ambos ciclos de la enseñanza secundaria.

2. Conocer las teorías pedagógicas que le permitirán desarrollar su labor docente.

3. Seleccionar métodos, técnicas y recursos didácticos adecuados y aplicarlos con eficacia en la enseñanza de la Ciencias.

4. Fortalecer la construcción de conocimientos científicos en sus estudiantes por medio de la utilización de demostraciones y experiencias de laboratorio.

5. Desarrollar destrezas para la observación sistemática y la investigación de los acontecimientos del aula.

6. Realizar un mejoramiento continuo de la práctica docente en el área de las Ciencias Básicas.

7. Aplicar evaluaciones adecuadas a la enseñanza de las ciencias.

8. Conocer las disposiciones de la ley 7600 sobre adecuaciones curriculares, para así, aplicarlas en el aula.

9. Desempeñar su tarea educativa con honestidad y en forma responsable.

10. Mejorar la enseñanza de las Ciencias mediante el conocimiento y la aplicación de técnicas y recursos que le sean óptimos y necesarios.

11. Lograr un profesional con habilidad en técnicas de comunicación interpersonal e interacción grupal.

12. Ofrecer a la sociedad costarricense un profesional en Ciencias que se mantenga actualizado en cuanto a los avances y tendencias del pensamiento científicotecnológico, educativo, cultural y social.

\section{Selección y organización de saberes}

Para lograr el perfil profesional y alcanzar los objetivos que se proponen para el plan de estudios, es necesario atender las sugerencias que surgieron del diagnóstico en cuanto a los aspectos epistemológicos. Se hace necesario que los profesores de ciencias 
experimenten una formación académica que les permita profundizar en los siguientes campos del conocimiento:

\begin{tabular}{|c|c|}
\hline Áreas del conocimiento & Contenidos requeridos \\
\hline Humanística & $\begin{array}{c}\text { Historia de la cultura. } \\
\text { Lenguaje y comunicación } \\
\text { Filosofía general } \\
\text { Realidad Nacional } \\
\text { Formación integral. (Repertorio) } \\
\text { Desarrollo sostenible } \\
\text { Artes y deportes } \\
\text { Trabajo comunal universitario }\end{array}$ \\
\hline Pedagogía y metodología & $\begin{array}{c}\text { Pedagogía } \\
\text { Didáctica general } \\
\text { Conocimientos básicos de currículum } \\
\text { Metodología y práctica profesional } \\
\text { Investigación educativa } \\
\text { Usos de los recursos didácticos. }\end{array}$ \\
\hline Psicología educativa y evaluación & $\begin{array}{c}\text { Psicopedagogía de los adolescentes } \\
\text { Atención a las necesidades educativas especiales } \\
\text { Principios de Evaluación y medición educativa }\end{array}$ \\
\hline Administración educativa & Administración curricular. \\
\hline Física & $\begin{array}{c}\text { Mecánica } \\
\text { Termodinámica } \\
\text { Electricidad y magnetismo } \\
\text { Óptica } \\
\text { Acústica } \\
\text { Fluidos } \\
\text { Física moderna } \\
\text { Meteorología } \\
\text { Astronomía }\end{array}$ \\
\hline
\end{tabular}




\begin{tabular}{|c|c|}
\hline \multirow{2}{*}{ Química } & Química general \\
& Química inorgánica \\
& Química orgánica \\
& Físico química \\
& Estequeometría \\
\hline \multirow{3}{*}{ Biología } & La vida, sus cambios y sus procesos. \\
& Los fenómenos de la herencia \\
& Evolución \\
& Biodiversidad general y de Costa Rica \\
& Ecología \\
& Recursos naturales \\
& Anatomía y fisiología humana \\
& Taxonomía \\
\hline Geología & Estructura geológica de la Tierra \\
& Geología de Costa Rica \\
& Desastres naturales \\
& Prevención de desastres \\
\hline Matemática & Cálculo diferencial \\
& Cálculo integral \\
\hline
\end{tabular}

Se proponen cursos existentes en la Universidad de Costa Rica, de manera que la puesta en marcha de esta propuesta, no genere nuevos cursos ni la necesidad de nuevos recursos.

En esta propuesta, los cursos de Educación para estudiantes que se forman como profesores de secundaria en cualquier especialidad, conocidos como núcleo pedagógico, no sufren cambios, ya que la Escuela de Formación Docente los tiene en revisión; pero se sugieren cursos o conceptos que se deben incluir en algún momento en la formación de este profesional.

Los cursos que ofrecen las diferentes unidades académicas involucradas en esta propuesta se ubican en una malla curricular que es flexible, se ajusta a las necesidades de los estudiantes y llena los requerimientos del plan de estudios, según los lineamientos 
establecidos por la Universidad de Costa Rica y su Vicerrectoría de Docencia. Igualmente, se incluyen áreas de la Acción Social, implícitas en el Trabajo Comunal Universitario (TCU) y la investigación cubierta en los cursos de investigación educativa. 


\section{Malla curricular}

Para cubrir los cursos propuestos para el plan de estudios se propone una malla curricular donde se ubican estos con sus respectivos requisitos y correquisitos.

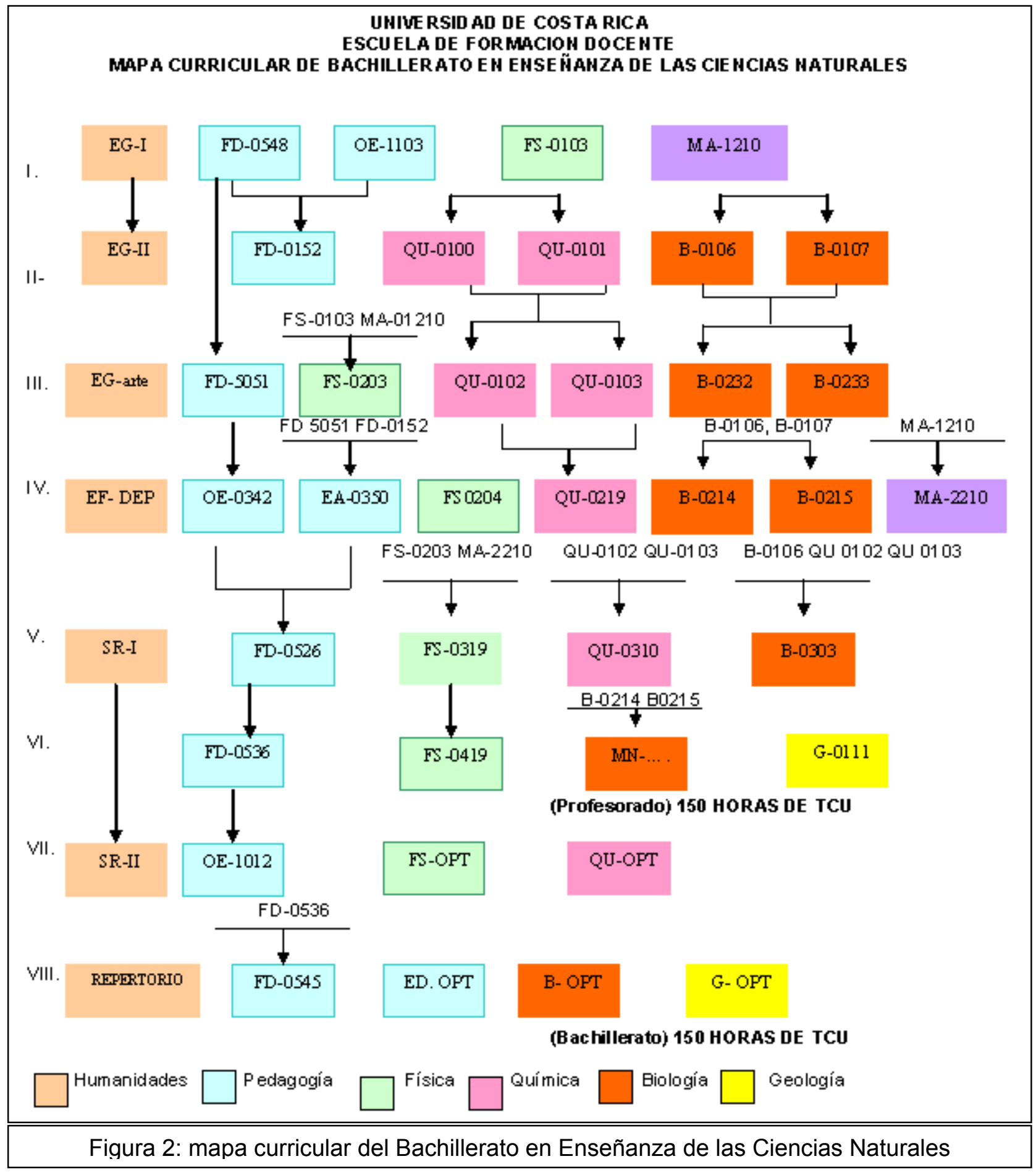


El esquema de la malla mencionada se aprecia en la figura 2 adjunta, que presenta un esquema tipo mapa curricular, siguiendo las condiciones de la malla establecida para esta propuesta.

En esta malla curricular se puede apreciar que los cursos se han ordenado de acuerdo con el área del conocimiento. La distribución de la misma obedece a la secuencia de los cursos requisitos, respetando que en cada ciclo se logre acomodar un máximo de 18 créditos.

Tal como se presenta la malla curricular, se puede dar flexibilización en los cursos optativos, en la ubicación de los cursos de cada área, de manera que el estudiante pueda matricular los cursos previo cumplimiento del requisito.

En cada una de las siguientes áreas se propone un curso optativo, el cual podrá ser seleccionado de la siguiente lista:

\begin{tabular}{|l|l|}
\hline \multirow{2}{*}{ Área de Educación } & $\begin{array}{l}\text { OE- } \\
\text { FD-0551 Seminario en la enseñanza de las ciencias. } \\
\text { FD-0130 Pedagogía y tecnología }\end{array}$ \\
\hline \multirow{4}{*}{ Área de Química } & $\begin{array}{l}\text { FS-0101 Fundamentos de astronomía } \\
\text { FS-0107 Fenómenos atmosféricos. } \\
\text { FS-0115 Fundamentos de oceanografía }\end{array}$ \\
\hline Área de Biología & $\begin{array}{l}\text { QU-0200 Química analítica } \\
\text { QU-0201 Laboratorio de Química analítica } \\
\text { QU-0110 Química general y biológica. }\end{array}$ \\
\hline Área de Geología & $\begin{array}{l}\text { B-0105 Introducción a la historia natural de Costa Rica. } \\
\text { B-0300 Problemas especiales en Biología } \\
\text { B 0122 Diversidad biológica de Costa Rica }\end{array}$ \\
\hline $\begin{array}{l}\text { G-0214 Geología general } \\
\text { G-0046 Geología de Costa Rica } \\
\text { G-0045 Desastres naturales. }\end{array}$ \\
\hline
\end{tabular}




\section{Referencias.}

Carvajal, C; otros. (1999) Orientaciones técnico-curriculares para el proceso de revisión del núcleo pedagógico. Escuela de Formación Docente. Universidad de Costa Rica. Costa Rica. Fotocopiado.

Centro de Evaluación Académica. (1991). Guía para la revisión y estructuración de planes de estudio. Universidad de Costa Rica. Vicerrectoría de Docencia. Costa Rica: Editorial de la Universidad de Costa Rica.

Delors, J. (1996) La educación encierra un tesoro. Madrid, España. Editorial Santillana.

Roldán, L. (2002) Informe final: Propuesta curricular para actualizar el plan de estudios ofrecido por la Universidad de Costa Rica para la carrera de bachillerato en la enseñanza de las ciencias naturales. Trabajo presentado para optar por el grado de Master en Planificación Curricular. Universidad de Costa Rica. San José. Costa Rica.

UNESCO. (1999). Conferencia Mundial sobre la Ciencia. República Dominicana. Recuperado el 15 de marzo de 2002, de Anuies.mx/anuies/revsup/res110

Vicerrectoría de Docencia. (1995). Políticas y normas curriculares para la actualización de planes de estudio. San José: Editorial de la Universidad de Costa Rica. 